\title{
A Topic-Driven Modular Approach to Engineering Education Delivery
}

\author{
http://dx.doi.org/10.3991/ijet.v8i1.2470 \\ R.A. Amarin ${ }^{1}$, O. O. Garibay ${ }^{1}$ and I. Batarseh*2 \\ ${ }^{1}$ University of Central Florida (UCF), Orlando, USA \\ ${ }^{2}$ Princess Sumaya University for Technology (PSUT), Amman, Jordan \\ *I. Batarseh is on Professional Development Leave at PSUT
}

\begin{abstract}
The interactive technical electronic book, TechEBook, currently under development at the University of Central Florida, provides a useful tool for engineers and scientists through unique features compared to the most used traditional electrical circuit textbooks available in the market. TechEBook has comprised the two worlds of classical circuit books and an interactive operating platform that can be run on mobile devices, laptops and desktops utilizing Java Virtual Machine operator. The TechEBook provides an interactive applets screen that holds many modules, in which each had a specific application in the self learning process.
\end{abstract}

In this paper the main goals and objectives behind the MeLearning concept will be identified. The paper will describe the differences between the traditional curriculum delivery and the MeLearning concept in details accompanied by the main tools used and a description of the different modules features.

Index Terms - Design Modules; Electrical Circuits; Interactive book; MeLearning; Practical Relevance Module; QuizMe; TutorMe.

\section{INTRODUCTION}

The interactive TechEBook consists of 16 chapters with a total of 75 sections representing typical content for the introductory circuit course at most universities in the world. Each section discusses a new theory and concept supported with examples and problems [1]. Different topics are presented in the discussion text that provides full understanding of the concept. At the end of each section, QuizMe Modules are provided to quiz the students' understanding of the section [2]. Also, TutorMe Modules are intended to help understand basic concepts in a step-by-step manner [3, 4]. Design Modules (DM) are intended to help students develop their ability in designing real life problems and to link the theories they study in books with real design challenges, while the Practical Relevance Modules (PRM) are set to enhance the students' capacity to think about real life problems and also to teach them how to relate the theories they have learned with practical applications. The SymCirc Symbolic Solver operates as a standalone web application, or as a utility integrated with the TechEBook, and allows users to get symbolic transfer functions and time domain expressions for any circuit with switches, which will enhance the user's capabilities to build a circuit, run a simulation, and obtain results in simple time domain functions [5].

\section{MELEARNING}

The $21^{\text {st }}$ century with the easy global access to the internet and the new You-Tube generation provides an unprecedented technological advantage and mandates that we change our education delivery; prepare our engineers to work across disciplinary boundaries and geographic boundaries in integrated teams that include designers, manufacturers, test personnel and others. Hence, we must change our pedagogical viewpoint and provide educational experiences that will match future needs [6].

Recognizing that the technology of the 21st century provides us with this unique opportunity to transform our education delivery system, this paper provides a modern, media-rich innovative approach to a topic-driven modular electrical engineering (EE) curriculum, which is called MeLearning. As noted by the NAE, "the delivery of instructional material is an important part of personalized learning". This paper introduces a paradigm shift, replacing the traditional EE course curriculum with topicdriven modules, offered outside the semester-limiting schedule. The instructor role will be that of a facilitator of learning and a mentor, providing higher level education interaction with the students on a regular basis. The MeLearning delivery system recognizes that every student approaches learning in a different way, some are selfmotivated and self-driven, some learn by example and problem solving, some need extra guidance, etc. A mediarich 'intelligent' modular delivery system becomes an important part of personalized learning and provides a closely articulated relationship between the outcomes and the level of achievement and assessment.

The MeLearning concept will take a major leap in educational delivery of engineering education and will facilitate learning at a pace specific to the learner without the constraints of a fixed time span - semester or quarter, currently specified in our curricula. The modular concept has several advantages over the traditional timeconstrained course delivery: (i) Creation of thematic and stand-alone modules will allow us to set different performance expectations for different themes in a semester-long course (ii) By allowing students to complete the module at their own pace, the modular learning caters to the diverse learning styles of students students who have non-auditory learning styles do not need to settle for 50-minute passive lectures to learn important concepts (iii) Because of (ii) above, performance levels tend to be consistently high even among a group of students with diverse learning styles, as opposed to a Gaussian distribution of performance levels 
in the traditional semester-constrained classes (iv) With a flexible pace of module completion, faculty-student interactions become more effective in the learning process; students needing longer times to complete a module will have more access to faculty whereas students who are blessed with learning styles that facilitate quicker learning are not unnecessarily slowed down by their slower-learning counterparts; in other words, flexible modular learning customizes the faculty-student interactions to suit the needs of the learner (v) Through the use of current technology, content creation, reuse, delivery, assessment, and evaluation can be made much more flexible and a learner centric educational system can be created.

\section{MAIN DRIVERS, GoALS AND OBJeCTIVES}

Attempts to move away from the traditional classroom delivery format, allowing for flexibility in the curriculum and developing dynamic curricula to infuse new ideas have been made at various institutions [7, 8]. Modular, flexible curricula have been proposed for computer science in Europe and in the US [9, 10]. The Synthesis Engineering Education Coalition, funded by NSF, studied the design, implementation and assessment of new approaches to undergraduate engineering education. Eight diverse universities were a part of this Coalition and their emphasis was on teamwork, communication, open-end problem formulation and solving multidisciplinary synthesis and examples of 'best practices' from industry. The Synthesis Coalition was a pioneer in developing multimedia courseware and evaluation and the effective use of education technology. They developed the National Engineering Education Delivery System (NEEDS) to facilitate discovery and sharing of engineering courseware. Connexions (cnx.org) is a site where authors can create, collaborate and share learning materials or modules. The MIT open courseware system is a very popular step in this direction, using You-Tube and modern technology to meet learning and education needs of diverse groups of students worldwide.

By providing a wide range of entry and exit points within the modular curriculum and continuous access to prerequisite resources, the student has greater flexibility in tailoring his/her individual program. The modular curriculum is more adaptable to the different needs of the program and several continuum paths can be identified for the student's choice. Redundancy of topics that exists in the traditional curriculum can be reduced significantly or eliminated for those who do not need the reinforcement; the modular framework allows accelerated learning for the high achievers. This can be translated into shortening the time to graduation and/or allowing time for design and research.

Such an evolutionary change in the education delivery approach requires organizational and institutional support and cooperation. Inherent dilemmas exist in the initial adoption of such a system and raise questions related to teaching loads, scheduling, time-constraints, tuition, 'ownership' of modules by departments etc. The College of Electrical Engineering and Computer Science (CECS) at the University of Central Florida (UCF) is fully in support of the TechEBook modular approach to the Electrical Engineering curriculum.

The main goals and objectives of the MeLearning initiative can be summarized as:

- Goals: The goals of this MeLearning concept are: (i) develop and adopt several modules for electrical engineering education delivery that provide personalized learning (ii) assess student performance via performance assessment at specific levels defined in any given module (iii) improve content delivery through continuous student feedback and appraisal .

- Objectives: (i) Develop several modules that correspond to two topics in the first electrical engineering course on electric circuits (ii) Assess the student performance within the MeLearning delivery program at specific bench-marking points and compare results with those of students in a control group (non-MeLearning electrical engineering students) (iii) Disseminate the results through publications and presentations.

\section{CReating Learning Materials And TeACHing STRATEGIES}

\section{A. MeLearning Approach vs. Traditional Curriculum Delivery}

In a MeLearning curriculum delivery system, the student will benefit through a more flexible selection of curriculum topics and a more efficient system of individual assessment. Table 1 presents a comparison of various educational issues in the MeLearning environment versus the traditional classroom delivery.

\section{B. Pace vs. Performance}

Arguably, one of the most educationally valuable aspects of a variable time sub-semester modular curriculum is the paradigm shift in fixed duration variable performance restriction that exists in the traditional academic environment. By inverting the dependence, a fixed performance - variable duration system may be created. In the MeLearning system, each course concept or topic is covered within the duration necessary to achieve mastery of the course material. This concept is illustrated in Figure 1. However, not all students will achieve mastery at an " $A$ " level, since the mastery threshold level is set by the module objectives.

The education benefits of this shift are available to all students, but impacts students at the extremes of the aptitude distribution more dramatically. Students who excel in the modular coursework may proceed to graduation at a quicker pace and more quickly continue to graduate school or find employment. At the other extreme, students who have difficulty in mastering engineering academic work may now take the time necessary to gain competency in contrast to being forced, in the semester system, to continue with addition subject material. All students who have their semesters disrupted by personal or work related absences will benefit as well. 
SHORT PAPER

A TOPIC-Driven MODUlar APproAch to ENGINEERING EDUCATION DELIVERY

TABLE I.

COMPARISON OF THE MELEARNING ENVIRONMENT TO THE TRADITIONAL CLASSROOM DELIVERY

\begin{tabular}{l|l|l}
\hline \multicolumn{1}{c|}{ Metric } & \multicolumn{1}{c}{ MeLearning } & \multicolumn{1}{c}{ Traditional Classroom } \\
\hline \hline Content Discretization & $\begin{array}{l}\text { Topic driven modules which are 3-9 hours in length } \\
\text { which are independent and assessable. }\end{array}$ & $\begin{array}{l}\text { Each current course consists of 45 hours (3 CH) of lecture } \\
\text { with multiple assessments that are averaged for a } \\
\text { combined grade. Students can fail to comprehend 30\% of } \\
\text { the material and still receive a grade of “C”. Courses } \\
\text { represent a coarse level of granularity. }\end{array}$ \\
\hline Curriculum Flexibility & $\begin{array}{l}\text { Students will be able to select modules which are } \\
\text { more finely defined and more diverse in topics. }\end{array}$ & Currently only 10 CHs of technical electives are available. \\
\hline Curriculum Reuse & $\begin{array}{l}\text { By using stored content, faculty can continually } \\
\text { improve topical content and use classroom time for } \\
\text { interactions with students. }\end{array}$ & $\begin{array}{l}\text { Every semester the same course content is delivered to a } \\
\text { new group of students. Lack of sufficient time for } \\
\text { improvement of quality. }\end{array}$ \\
\hline Remediation & $\begin{array}{l}\text { Students who do not master a topic must repeat the } \\
\text { topic through the use of existing content }\end{array}$ & $\begin{array}{l}\text { Students are on their own, or they must repeat an entire } \\
\text { course. }\end{array}$ \\
\hline Student Tracking & Software Based & Manual or MyUCF \\
\hline $\begin{array}{l}\text { ABET (Accreditation Board for } \\
\text { Engineering and Technology, } \\
\text { Inc.) }\end{array}$ & $\begin{array}{l}\text { Online or web assisted assessment meets the need for } \\
\text { individual assessment and accumulation of student } \\
\text { performance. }\end{array}$ & $\begin{array}{l}\text { Group in-class exams require considerable effort to extract } \\
\text { individual assessment data as needed for ABET. }\end{array}$ \\
\hline
\end{tabular}

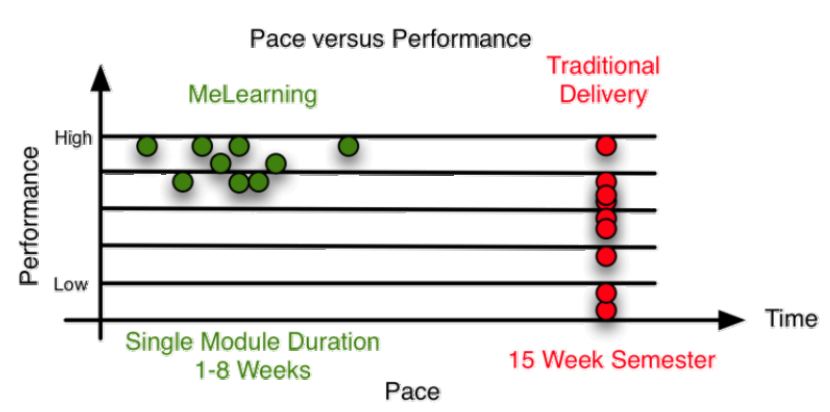

Figure 1. Shift in educational paradigm on pace and performance

\section{Description of Module Features}

\section{A. TutorMe Tool}

An interactive, individualized, electrical circuits tutoring (TutorMe) tool is developed to help students learn key concepts in electrical circuit analysis methods. This tool will guide the student through a step-by-step analysis of common electrical circuit problems. In each step, the tool prompts the student to enter a formula that best describes the current step in the analysis method. Then, the tool responds with personalized feedback that is dependent on the user's formula. Through the feedback process, the tool targets all lexical and analytical errors in the user input. The tool compiles a list of errors and warning messages that are reported back to the student. Trials and interactive elaboration on errors allow the student to better understand the targeted concept in circuit analysis. The modular and template-based design for the tool allows the instructor to add and modify key problems.

Since it is difficult for a professor to effectively evaluate the comprehension level of each student, highly personalized instructor-student tutoring could result in a delay of class material. With the TutorMe tool, students will be able to solve problems and obtain the same type of specific feedback they would receive from the class instructor. The TutorMe will not only inform the students if their input to a question was right or wrong, it will also specify what type of error was made in the symbolic equation. More importantly, each student learns at his/her own pace, based on their individual learning styles.

Figure 2 shows the main screen of the TutorMe tool. The user selects the circuit example of interest from the dropdown menu which is designed such that the instructor can add circuits easily. The user then can walk through the example step by step using the navigation buttons. Each step provides the needed description and the associated a circuit image, and if applicable, an input box for the user equation is displayed. The output box at the bottom of the TutorMe window shows the results of the parsing and analysis operation. This box mainly displays all errors, warnings, and success messages generated during processing. Finally, a screen that allows the user to solve the circuit numerically is given as an option for the user once the last step of the problem is passed.

\section{B. QuizMe Tool}

QuizMe is an advanced quizzing tool to assess the student's understanding of the electrical circuit concepts and help instructors to evaluate the student's performance and track their improvement. The tool is adaptive in the sense that the question's difficulty level adapts to student performance, the more the student scores the more difficult the questions get. Finally, the module is designed around the self-learning theme, it displays instant feedback to the students along with the correct answers so that the students can use the tool by their own to practice and improve. The QuizMe main interface is shown in Figure 3.

The users of the QuizMe are students and instructors. Instructors can design quizzes with a variety of built-in options, such as number of attempts, adaptive testing, and

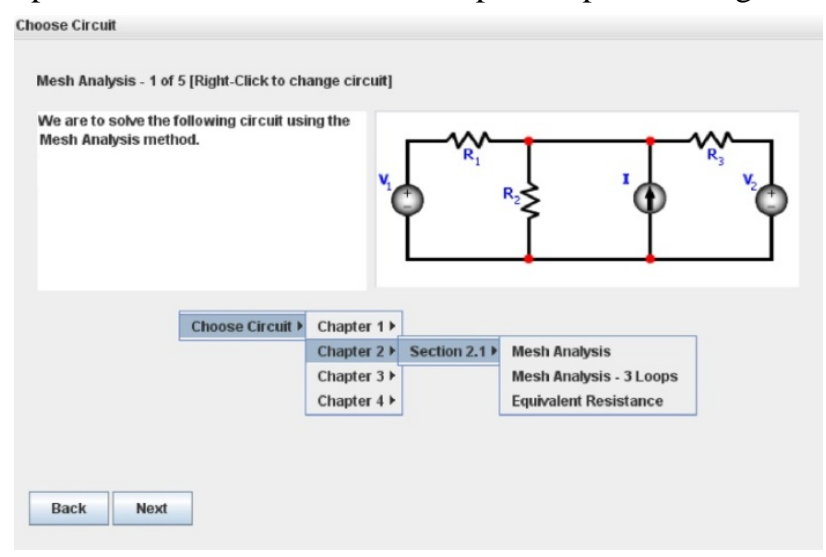

Figure 2. TutorMe main screen 


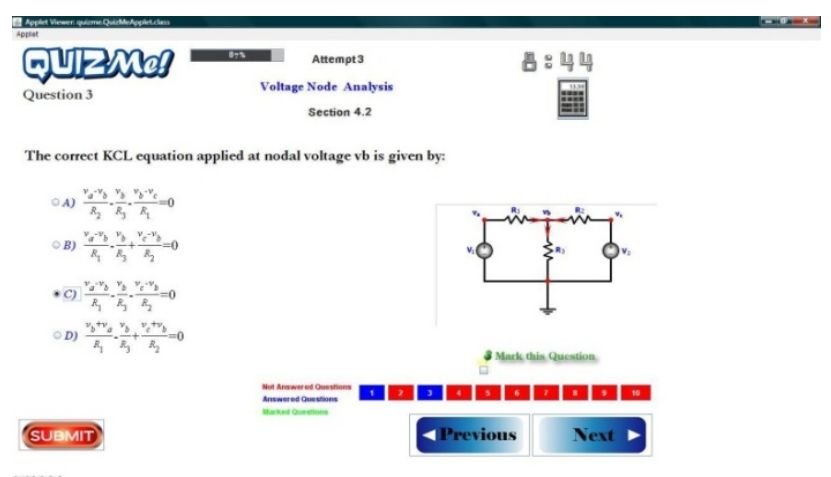

Figure 3. QuizMe main interface

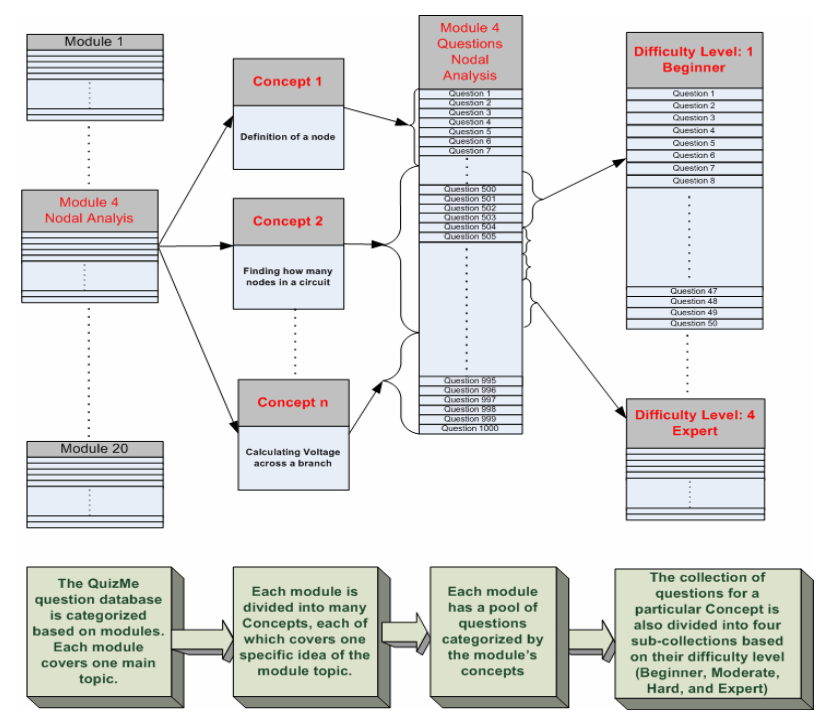

Figure 4. QuizMe questions pool

questions selection criteria. They can also add, remove, or update questions and images, as well as decide the length of the quiz. Finally, the instructors can access the students' records, grades, and answers to evaluate their performance, and generate performance and grade report for their classrooms. Figure 4 shows a detailed diagram for the QuizMe questions pool, it shows how the QuizMe questions in a module are divided into sections, with each section containing set "Concepts" based on specific ideas introduced in the module. Each concept in turn contains sets of questions grouped based on the difficulty level; in this example each concept contains questions in four increasing levels of difficulty. The QuizMe tool is able to access this large pool of questions and filter them to create quizzes suited to the student's needs and ability.

\section{Design Modules Tool}

Most electrical engineering books tend to be abstract and have very limited design examples and problems. The Design Module (DM) tool will be used to help students understand the topic being discussed and to make sure they have a complete understanding of the theory. It will be a collection of electrical engineering problems relevant to the module and will support the theory with better insight and clarity. Each design tool will have four design problems that will have different difficulty levels. The idea is to allow students at all academic levels to be exposed to the same electrical engineering concepts at the difficulty level they can comprehend. The purpose is to cover large range levels of student understanding.
The DM includes solving for different circuit parameters to satisfy a certain condition. This will improve the students' ability to design the circuit in many different ways to enhance their engineering sense.

\section{Practical Relevance Tool}

The objective of this tool is to enable students to interactively learn engineering concepts through practical applications of the theory. The practical relevance exercises will be dynamic with animation features and the ability for students to enter various values for formulas, which in turn will be dynamically used to convey a variety of results for the specific topics. The Practical Relevance Tool provides engineering students with an understanding of the practical application of the abstract theories by solving real-world examples and problems. Moreover, this tool will be highly interactive with illustrated animations. The tool will present real-world applications, relate the application to the scientific theory being studied, relate the application to a real product in the market, and provide an interactive applet to show how the application works under different parameter variations.

Almost all of today's consumer electronics products on the market are designed based on a technical area in the field of electrical engineering. These areas include electrical circuits, electromagnetics, analogue electronics, controls, digital electronics, digital signal processing, digital and analogue filters, digital and analogue communications, computer architecture, sensors, networking, power electronics, power systems, optics and photonics, lasers, antennas and satellites, machines, and drives. Ultimately, the electrical engineering concepts that use the technical areas listed above are used to develop new technology and products: cell phones, MP3 players, CD players, DVD players, TVs, radios, computers, calculators, displays, cars, planes, trains, motorcycles, ovens, refrigerators, dishwashers, microwaves, power outlets, air conditioners, power supplies, lamps, and lawnmowers. Each of the above products can be used to generate many real-world examples that use the technical areas mentioned above.

One example of a practical relevance scenario is the voltage amplifier circuit, which is mainly used in consumer electronics and communication applications such as radios, TVs, cell phones, satellites, VCRs and DVDs. In this practical relevance example for the meshcurrent analysis method, a typical voltage amplifier circuit that may be used in radios to amplify a weak voltage signal would be discussed and key findings, i.e. voltage gain will be computed in detailed steps.

\section{E. Symbolic Circuit Solver Tool}

The Symbolic Circuit Solver (SymCirc) is one major component of the TechEBook currently being developed. The main objective of including this module is to provide engineering students in the electrical circuit classes with a more robust understanding of the electrical network theory through building and designing real time examples.

The SymCirc Symbolic Solver provides all simulation results as Time domain expressions composed of the basic functions that exclusively include exponentials, sines, cosines and/or $t$ raised to any power. One big motivation for taking the symbolic simulation route is the fact that for circuits with switching components, convergence problems are avoided, since elements are represented as 


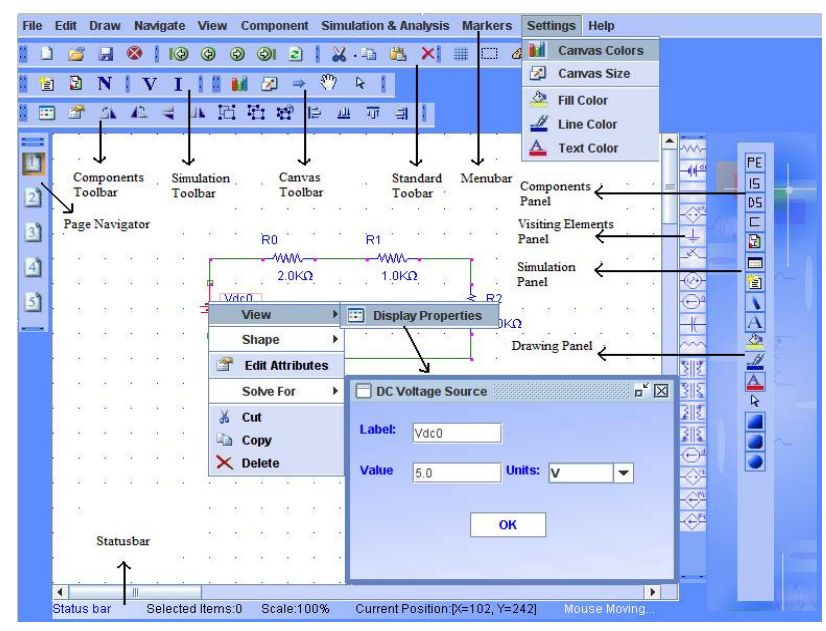

Figure 5. Graphical user interface front end for SymCirc

symbols rather than numbers. Also subsequent simulation of the same circuit with different parameter values is much faster, due to the fact that the system of equations representing the circuits need not be solved more than once. The SymCirc develops a versatile symbolic based linear circuit with a switches solver. The solver works by accepting a Netlist and the element that the user wants to find the voltage across or current on, as input parameters. Then it either produces the plot or the time domain expression of the output. Frequency domain plots or Symbolic Transfer Functions are also produced. The solver gets its input from a Web-based GUI circuit drawer developed at UCF. Typical simulation tools that electrical engineers encounter are numerical in nature, that is, when presented with an input circuit they iteratively solve the circuit across a set of small time steps. The SymCirc graphical user interface front end is shown in Figure 5.

\section{CONCLUSION}

The MeLearning project will take a major leap in engineering education and will facilitate learning at a pace specific to the learner without the constraints of a fixed time span - semester or quarter, currently specified in our curricula. This paper, addresses the TechEBook objectives and the advantages of the MeLearning concept. The main tools used and a description of the different modules features are also introduced.

The main purpose of the TutorMe technique is to provide a well defined step-by-step interactive problem solving that will help in testing the user understanding of key concepts in each main section presented by the TechEBook. While the main purpose of the QuizMe technique is to give a well established and fair feedback, and give different ways on how to improve in any certain subject. The PRM and DM techniques are developed to give a well understanding and a fair knowledge about relating theories to real world applications, and to enhance the engineering sense within students. As for the SymCirc Symbolic Solver, it helps users understand the ultimate nature of circuits as Linear Time Invariant systems with a finite dimensional basis in the solution space by providing all the simulation results as time domain expressions composed of the basic functions. SymCirc will maximize the students learning experience, in their self-learning process (me-applying, me-designing).

\section{REFERENCES}

[1] Issa Batarseh, Ghaith Haddad, Rawad Haddad and Rashad Oreifij, 'Interactive Electronic Book Operating Systems And Methods,' United States Patent 20080222552.

[2] Ruba A. Amarin, Feras Batarseh and Issa Batarseh, 'Adaptive Electronic Quizzing Method for Introductory Electrical Circuit Course', International Journal of Online Engineering, 2009.

[3] Ghaith Haddad, Gustavo Gamboa and Issa Batarseh, 'Interactive Electrical Circuit Tutoring Tool. eTutor,' 2008 ASEE Southeast Section Conference, Memphis, TN, April 2008.

[4] Ruba A. Amarin, Issa Batarseh, 'eTutor - An Interactive Module for Electrical Engineering Curriculum', ASEE Conference, Philadelphia, PA, 2011.

[5] Ehab Shoubaki, Ruba Amarin and Issa Batarseh, ' Java Based Symbolic Circuit Solver For Electrical Engineering Curriculum', IEEE EDUCON Conference, Marrakesh, Morocco, April 2012.

[6] 2. Paradigm Shift in Engineering Education, J. Bourne, A. Brodersen, The Influence of Technology on Engineering Education, Ed. J. Bourne, A. Brodersen and M. Dawant, CRC Press, 1995 .

[7] Developing the Credit-based Modular Curriculum in Higher Education, Challenges, Choice and Change, Mike Betts and Robin Smith, Routledge, 1998.

[8] Modular Curriculum: An Alternative Approach to Engineering Education, M. Moussavi, ASEE Frontiers in Education Conference, 1997.

[9] A Modular Curriculum in Computer Science, UNESCO, 1994.

[10] Towards an Active Curriculum for Computer Science, S. Kabicher, M. Derntl and R. Motschnig, Proceedings of World Conference on Educational Multimedia, Hypermedia and Telecommunications, 2008.

\section{AUTHORS}

Ruba A. Amarin is with the University of Central Florida, Orlando, FL 32816 USA (e-mail: ramarin@mail.ucf.edu). Ruba received the B.S. degree in Electronics Engineering from the Princess Sumaya University for Technology, Amman-Jordan in 2004; she afterwards received her M.S. degree in Electrical Engineering, Tele-Communication track from the University of Central Florida, Orlando in 2006. In 2010, Ruba received her Ph.D. in Electrical Engineering, conducting research in Satellite and Remote Sensing and her M.S. degree in Industrial Engineering specializing in Engineering Management. She has several U.S. patents pending in the power electronics and smart grid fields and more than 40 refereed journal and conference publications.

O. Garibay is with the University of Central Florida, Orlando, FL 32816 USA (e-mail: Ozlem@ucf.edu).

Issa Batarseh is currently on professional development leave at the Princess Sumaya University for Technology (PSUT) (e-mail: issa.batarseh@psut.edu.jo). He received the B.S. degree in Computer Engineering and the M.S. and Ph.D. degrees in Electrical Engineering from University of Illinois, Chicago, in 1983, 1985, and 1990, respectively. He was a Visiting Assistant Professor of Electrical Engineering at Purdue University, Calumet City, IN, from 1989 to 1990 before joining the Department of Electrical and Computer Engineering at the University of Central Florida, in 1991. He has more than 14 U.S. patents, and more than 50 refereed journal and 200 conference publications.

This work is partially funded by NSF under CCLI Award Number: 0837364. Received 02 January 2013. Published as resubmitted by the authors 27 february 2013. 